\title{
TRAITEMENT DE LA MALADIE DU SOMMEIL DU BETAIL AU DIMIDIUM BROMIDE
}

par le Docteur M. BLOMMAERT

Sur demande de M. le Directeur de la Compagnie des Grands Élevages congolais à Biano, nous avons essayé le Dimidium Bromide contre la maladie du sommeil du bétail. Ces essais ont débuté le 19 Mai 1947 pour prendre fin le 24 Février 1948.

Des observations individuelles ont été faites sur 20 bêtes et sur 91 autres il fut procédé à des traitements en masse.

\section{Essais individuels.}

Les 20 premières bêtcs trouvécs trypanosécs furent conccntrées cn un endroit indemne de glossines, marquées d'un numéro d'ordre et soumises à des examens cliniques et microscopiques.

\section{$1^{\circ}$ Symptômes et diagnostics.}

Nous àvons trouvé :

a) 3 bêtes infectées de Tryp. Congolense et Vivax, 8 de Tryp. Congolense seul et 9 de Tryp. Vivax seul.

b) 6 bêtes dont l'état général était manvais, 2 dont l'état était plus ou moins bons et 12 en bon état général.

c) Quant aux ganglions, 4 bêtes les avaient fortement et 6 légèrement gonflés, 10 bêtes les avaient normaux.

La symptomatologie démontre done que les bêtes sont dans des stades différents de la maladie.

\section{$2^{\circ}$ Technique.}

a) Nous avons employé 13 fois le produit MAY and BAKER (formule non mentionnée) et 9 fois celui de Burrougs Wellcome (formule : 2 : 7 Diamino9-Phényl-10 Méthyl Phénantridinium Bromide).

b) La concentration varie entre 0,5 et $3 \%$.

c) Nous avons fait 21 injections intraveineuses dans la jugulaire sur l'animal couché, une injection sous la peau, dans le fanon.

d) Pour 17 cas la dose était de 1 mgr. par kilo de poids vif et pour 50 cas de 2 mgr. par kilo de poids vif.

e) Nous avons fait 10 à 22 examens de contrôle pour chaque sujet. Le premier a eu lieu 24 à 48 heures après l'injection, les autres à une fréquence hebdomadaire.

f) Tenant compte qu'une verminose, tout en affaiblissant la résistance naturelle, peut influencer la convalescence d'une autre maladie et que les 
symptômes de la verminose peuvent être confondus avec ceux de la trypanosomiase, nous avons traité à l'antivermineux chaque sujet atteint de cette maladie.

\section{$3^{\circ}$ Discussion:}

a) Marque duproduit: Nous ne voyons pas de différence importanté entre l'activité des deux produits, les deux ont donné une rechute à la dose de 1 mgr. par kilo de poids vif.

b) Concenirration : Les différentes concentrations employées 'n'ont donné aucun inconvénient. Il serait impossible de préconiser l'une ou l'autre. Personnellement nous avons adopté la concentration de $1 \%$ poür des raisons techniques.

c) Mode d'emploi : L'injection intraveineuse dans la jugulaire sur l'animal couché a donné des bons résultats. L'injection sous-cutanée dans le fanon a donné une forte réaction locale. Il s'est produit un gonflement de la grosseur d'une tête humaine, qui a persisté 2 mois. Le gonflement s'est résorbé lentement sans suppuration (application hebdomadaire d'onguent mercuriel). A l'heure actuelle il persiste une sclérose. La bête malade n'a plus récidivé et se trouve en bon état de santé.

d) Dose : A la dose de 1 mgr. par kilo de poids vif nous avons constaté 2 rechutes (les $N^{\text {os }} 9$ et 17 ) sur 17 cas.

Le No 9 était déjà récidiviste au premier examen, il fut traité antérieurement à l'émétique. Après une dose de Dimidium Bromide un peu supérieure à $1 \mathrm{mgr}$. par kilo de poids vif, il a récidivé une deuxième fois 74 jours après le traitement. Après la deuxième injection à dose double, nous n'avons plus trouvé de trypanosomes, la bête est en bon état général actuellement.

Le $\mathrm{N}^{\circ} 17$ a montré des trypanosomes vivax au premier examen des tryp. congolense 37 jours après le traitement.' Il est probable que déjà au premier examen la bête était atteinte d'une infection mixte à Congolense et à Vivax, mais que les Tryp. congolense sont passés inaperçus ou ne se trouvaient pas dans le sang périphérique lors du premier examen. Comme tout le bétail traité a pâturé duns des endroils où jamais la trypanosomiase n'a été constatée, il est improbable qu'il y ait eu une infection après le premier traitement. Le bœuf a été traité une deuxième fois à dose double. Nous n'avons plus trouvé de trypanosomes après le traitement, et la bête est en bon état. A partir de ce jour chaque bête a été traitée à la dose de 2 mgr. par kilo de poids vif.

e) Examen de contrôle : Il n'a plus jamais été trouvé un seul trypanosome pour 16 bêtes traitées. Hormis les deux rechutes mentionnées cidessus; nous avons encore trouvé dans deux cas des trypanosomes 24 à 48 heures après le traitement.

Notamment : le $\mathrm{N}^{\circ} 15$ un Tryp. indéterminable (autolyse?)

le No 20 un Tryp. vivax.

Après 48 heures et jusqu'à présent, il n'a plus été possible de trouver de trypanosomes chez ces deux boufs. 
Nous avons encore pu constater le même phénomène dans deux autres cas non mentionnés dans les essais individuels.

Il faut en conclure que probablement le médicament agit lentement et que trouver des trypanosomes 48 heures après l'injection ne veut pas dire qu'il ' s'agit d'un échec. Il serait intéressant de savoir combien de temps le médicament reste dans l'organisme. Pour cette raison, uous avons autopsié un bouvillon de 2 ans fortement anémié par la verminose 40 heures après l'injection. A l'œil nu nous n'avons trouvé aucune trace du médicament.

Les tissus conjonctifs n'avaient pas la couleur du médicament, l'urine avait l'aspect normal, le cœur, le foie, la rate et les reins ne présentaient rien que de l'anémie sans aucun changement pathologique qui pourrait être imputé au Dimidium Bromide.

f) Réactions générales: Aucune réaction générale grave due à l'injection n'a été constatée, si ce n'est dans un cas en dehors des essais.

Une vache de 8 ans atteinte de trypanosomiase aiguë à congolense (décu. bitus, pouls rapide, respiration accélérée) a recu 2 mgr. par kilo de poids vif à $1 \%$ dans la jugulaire. Elle a succombé quelques instants après l'injection. Nous en concluons qu'en' pareil cas, il serait peut être utile d'injecter en un point plus éloigné du cour afin que le mélange avec le sang se fasse plus lentement : par exemple dans une veine de l'oreille.

g) Convalescence : Nous constatons que le bétail mis dans une bonne pâture après un traitement au Dimidium Bromide reprend rapidement.

h) Mortalité : Il y a eu une mortalité 16 jours après le traitement. Il s'agissait d'un bouf de trait en cachexie par la verminose ( $N^{\circ} 13$ ). Il a subi trois examens de contrôle avant sa mort, tous les trois étaient nègatifs.

\section{1. - Traitements en masse.}

En saison sèche 1947, suite à une pénurie de pâturages, plisieurs troupeaux de bœufs et de bouvillons ont dû aller pâturer dans des endroits où l'on trouve parfois des glossines.

91 bètes trouvées atteintes de trypanosomiase ont étẻ soumises à un traitement au Dimidium Bromide : May and Baker, 2 milligrammes par kỉo de poids vif $1 \%$ I.V. à partir d'Octobre 1947.

40 ont été traitées en Octobre, 18 en Novembre, 15 en Décembre 1947 et 18 en Janvier 1948 .

Un infirmier microscopiste indigène a fait des examens de contrôle en Novembre et Décembre 1947 ainsi qu'en Janvier 1948.

Un examen a èté fait par l'auteur en Février 1948.

Pour des raisons expérimentales, 10 bêtes ont reçu une dose de Dimidium Bromide de 2,5 à 3 milligrammes par kilo de poids vif.

\section{DISCUSSION.}

a) Réactions : A la dose de 2 milligrammes par kilo de poids vif, nous n'avons pas remarqué de réactions immédiates ni tardives. 
A la dose de 3 milligrammes par kilo de poids vif, une bête en bon état général a fait un choc passager : mouvements désordonnés de la tête et des membres, respiration lente et pénible, pouls rapide. Cela n'a guère duré plus d'une minute, la bête s'est relevée et peu après paraissait normale.

Les autres bêtes injectées à raison de 2,5 à 3 milligrammes par ki lo de poids vif ont parfaitement supporté la dose.

b) Pholosensibilité : La plupart des bêtes traitées ont un derme fortement pigmenté. Ún seul bouvillon, à la robe Héreford, a fait une photodermatite peu grave. La dermatite s'est localisée exclusivement, aux parties non pigmentées de la peau. L'animal n'en a apparemment pas souffert beaucoup. En dehors de ce cas, nous n'avons pu constater de la photodermatite chez aucune bête ayant un derme non pigmenté. Remarquons que nous avons constaté de la photodermatite sur un bouvillon d'un autre troupeau n'ayant pas été traité au Dimidium Bromide.

c) Rechules : Lors du contrôle, nous avons constaté 3 cas de Tryp. congolense, aucun de vivax. C'était des bêtes traitées entre le 15 Octobre et le 20 Décembre 1947. Il est évidemment'impossible d'affirmer' s'il s'agit de véritables rechutes ou de réinfections, car le bétail traité a pâturé dans des endroits où l'infection n'est pas exclue $100 \%$.

d) Convalescence : Comme dans les essais individuels, nous avons pu constater une rapide amélioration de l'état général.

e) Mortalité : Aucune mortalité due à la maladie du sommeil n'est survenue.

\section{III. - Résumé.}

$1^{\circ}$ Le Dimidium Bromide injecté à la dose de 2 milligrammes par kilo de poids vif donne des résultats plus sûrs qu'à 1 milligramme. Il peut être dangereux à 3 milligrammes par kilo de poids vif.

$2^{\circ}$ Chez des bêtes gravement malades, il faut user de beaucoup de précautions.

$3^{\circ}$ Un examen positif 48 heures après le traitement ne veut pas dire nécessairement qu'il s'agisse d'un échec.

$4^{\circ}$ Les rechutes sont peu fréquentes. Il est à remarquer que parmi les 5 rechutes constatées par l'auteur, il ne s'agit que de Tryp. congolense. Le médicament semble donc plus actif contre les Tryp. vivax.

$5^{\circ}$ La convalescence est rapide.

$6^{\circ}$ Les réactions de photosensibilité sont possibles.

$7^{\circ}$ L'injection sous-cutanée donne une forte réaction locale. 
TRAITEMENT DE LA MALADIE DU SOMMEIL DU BtTaIL

\section{IV. - Conclusion.}

Les essais relatés ci-dessus permettent d'affirmer que le Dimidium Bromide constitue incontestablement un enrichissement très intéressant de la thérapeutique de la maladie du sommeil du bétail.

Il a les avantages suivants:

$1^{\circ}$ il ne faut qu'une seule intervention;

$2^{\circ}$ peu de réactions ou de suites désagréables sont à craindre;

$3^{\circ}$ les rechutes sont peu fréquentes;

$4^{\circ}$ la convalescence est rapide. 